\title{
An Agent-Based Distributed Coordination Mechanism for Wireless Visual Sensor Nodes Using Dynamic Programming
}

\author{
Johnsen Kho, Long Tran-Thanh, Alex Rogers and Nicholas \\ R. JENNINGS
}

School of Electronics and Computer Science, University of Southampton, Southampton, SO17 $1 B J, U K$

Email: $\{j k 05 r, l t t 08 r, a c r, n r j\} @ e c s . s o t o n . a c . u k$

\begin{abstract}
The efficient management of the limited energy resources of a wireless visual sensor network is central to its successful operation. Within this context, this article focuses on the adaptive sampling, forwarding, and routing actions of each node in order to maximise the information value of the data collected. These actions are inter-related in a multi-hop routing scenario because each node's energy consumption must be optimally allocated between sampling and transmitting its own data, receiving and forwarding the data of other nodes, and routing any data. Thus, we develop two optimal agent-based decentralised algorithms to solve this distributed constraint optimization problem. The first assumes that the route by which data is forwarded to the base station is fixed, and then calculates the optimal sampling, transmitting, and forwarding actions that each node should perform. The second assumes flexible routing, and makes optimal decisions regarding both the integration of actions that each node should choose, and also the route by which the data should be forwarded to the base station. The two algorithms represent a trade-off in optimality, communication cost, and processing time. In an empirical evaluation on sensor networks (whose underlying communication networks exhibit loops), we show that the algorithm with flexible routing is able to deliver approximately twice the quantity of information to the base station compared to the algorithm using fixed routing (where an arbitrary choice of route is made). However, this gain comes at a considerable communication and computational cost (increasing both by a factor of 100 times). Thus, while the algorithm with flexible routing is suitable for networks with a small numbers of nodes, it scales poorly, and as the size of the network increases, the algorithm with fixed routing is favoured.
\end{abstract}

\section{INTRODUCTION}

Wireless sensor networks, composed of locally batterypowered sensor nodes that wirelessly communicate and route information sampled from the environment through the network to a base station, are receiving significant multi-disciplinary research interest from institutions around the globe. Due to their flexibility, cost effectiveness, ease of deployment, scalability, and dynamic coverage, wireless sensor networks are becoming increasingly prevalent in a wide variety of applications, including environmental and habitat monitoring [1, 2, 3], structural health surveillance [4], smart buildings [5], and other health related applications [6].

The rapidly increasing computational power of the nodes deployed within such networks has allowed them to perform ever more sophisticated tasks, and recently, wireless visual sensor networks (WVSNs), whose nodes consist of spatially distributed smart camera devices, have received increasing attention within the research community. Each node within these networks has its own image sensor, image processing, storage, communication, and limited power units (see Fig. 1 for an example of a wireless smart camera mote), such that it is capable of performing basic capturing and compression of visual data before relaying it to a base station, in a multi-hop fashion, to be processed, analysed, or fused into some form more useful than the individual data (e.g. to reconstruct the entire surveillance area covered by the network). WVSNs are therefore intended for distributed image acquisition 


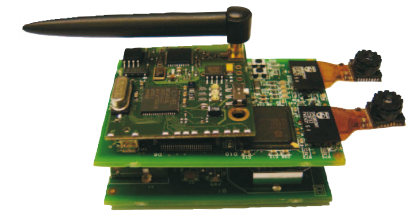

(a)

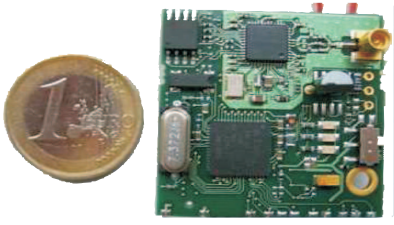

(b)
FIGURE 1. (a) Wireless smart camera mote (taken from [13]) which is battery-powered and equipped with two VGA colour image sensors, SIMD (Single Instruction Multiple Data) image-analysis processor and 8051 micro-controller, a dual port RAM with $128 \mathrm{~KB}$ memory, and a ZigBee IEEE 802.15.4 communication module, as shown in (b).

(in a variety of applications such as object tracking in battlefields $[7,8]$, unattended area surveillance $[9,10]$, and other security related applications) over large and possibly hostile environments and, as such, are required to operate for extended periods of time with minimal human intervention. However, the increased computational power of the nodes within a WVSN compared to those typically deployed within a conventional wireless sensor network, the large amounts of visual information that they collect, and the high energy cost of wirelessly communicating this information through the network, mean that efficient energy management remains a key challenge in these networks $[11,12]$.

To date, efficient power management has been addressed through (i) hardware and (ii) software. Within the former, advances in chip manufacture have successfully reduced the power consumption of nodes, helping to improve their longevity, and, in turn, the network's lifetime $[14,15]$. Moreover, in some sensor network applications, an additional facility is available to ameliorate the energy problem by using rechargeable batteries and energy harvesting technologies such as solar panels, wind turbines, piezo-electric harvesters, or other transducers $[16,17]$. From the latter perspective, however, work has addressed the two main actions that such sensor nodes can vary in order to make their energy management more efficient: (i) their sampling rate (i.e. how much visual data they acquire) and (ii) their communication of data capabilities (those include selecting the most energy efficient path between the node and the base station given that the nodes may have different energy constraints and communication costs).

In particular, recent work has explored decentralised coordination algorithms that enable the nodes to autonomously adapt and adjust their sampling and communication behaviour. This coordination is computationally expensive since the sampling and communication decisions are inter-dependent in a multi-hop routing scenario. This is because each node's energy consumption must be optimally allocated between sampling and transmitting its own data, receiving and forwarding the data of other nodes, and routing any data. In such a setting, the choices of one node can potentially affect all other nodes in the network. As such, much of this work has developed approximate decentralised algorithms, or has used simple heuristic algorithms that allow the nodes to make local decisions to improve the overall performance of the network (see Sect. 5 for more details).

While such approaches have proved valuable in the context in which they were developed, the move to WVSN means that there is now the possibility of deploying complete algorithms that can optimally maximise the overall effectiveness of the dynamic network through distributed computation, rather than local heuristics. It is this challenge that we address, and to this end, we adopt an agent-based approach in which each node is represented as an autonomous agent (with private information regarding its own state), and the complex, inter-connected, and rapidly changing network, as a multi-agent system in which the individual agents need to coordinate their activities cooperatively in a distributed manner towards achieving the system-wide goals (that is, each agent is capable of reactive, social, and goal-directed behaviour). Within this context, each node must be able to decide for itself whether or not to perform an action requested by another node. This is different from the node being a passive distributed object which encapsulates some private states and has public methods corresponding to operations that other nodes are always allowed to perform on these states.

Against this background, in this article, we develop a novel distributed algorithm that varies each node's inter-dependent sampling, transmitting, and forwarding rates (or actions) to ensure all nodes in a network focus their limited resources on maximising the information content of the data collected at the base station. The algorithm is complete meaning that it is capable of finding an optimal solution, if one exists, otherwise it correctly reports that no solution is feasible. We first assume that the route by which data is forwarded to the base station is fixed (either because the underlying communication network is a tree, or because an arbitrary choice of route has been made), and show that the resulting algorithm is scalable (running on hundreds or even thousands of nodes in real-time) and has minimal memory requirements. By utilizing distributed dynamic programming techniques, each node makes computationally tractable and optimal local decisions regarding its integration of actions by only exchanging a small number of coordination messages with its parent and children nodes.

Using the same technique to extensively truncate the search space of potential resource allocation decisions, we then extend the fixed route algorithm to deal with flexible routing, in which each node not only makes optimal decisions regarding the inter-related sampling, transmitting, and forwarding actions, but also determines the optimal route by which each item of 
sensed data should be forwarded to the base station. To ground and evaluate these algorithms, we empirically evaluate them and show that they represent a tradeoff in optimality, communication cost, and processing time. In more detail, we show that when deployed on sensor networks with loopy topology (i.e. where data can follow multiple paths to the base station), the algorithm with flexible routing is able to deliver approximately twice the quantity of information to the base station compared to that of the algorithm using fixed routing. However, this gain comes at a considerable communication and computational cost (increasing both by a factor of 100 times). Thus, while the algorithm with flexible routing is suitable for networks with a small numbers of nodes, it scales poorly, and as the size of the network increases, the algorithm with fixed routing is favoured.

The remainder of this paper is organized as follows. In Sect. 2 we state the formal model of our distributed constraint optimization problem (i.e. the interrelated adaptive sampling, transmitting, forwarding, and routing problem). In Sect. 3 we detail our two novel algorithms and show how we find the optimal local allocation decisions. Our experimental results are presented in Sect. 4. We present previous work in this area in Sect. 5 and we conclude in Sect. 6.

\section{PROBLEM DESCRIPTION}

Here, we formalise the generic inter-related adaptive sampling, transmitting, forwarding, and routing problem that we face. To this end, let $n$ be the number of visual sensor nodes within a WVSN system and the set of all nodes (or agents) be $I=\{1, \ldots, n\}$. Each node $i \in I$ can sample at $s_{i}$ different rates over a period of time. Its set of possible sampling (or frame) rates is denoted by $C_{i}=\left\{c_{i}^{1}, \ldots, c_{i}^{s_{i}}\right\}$. Specifically, each element of this set, $c_{i}^{j}$, is a positive integer that describes the number of samples that the node takes during any specific time interval.

Each node has private information regarding the information content of the samples that it acquires, and this is represented by an array of 2-tuples $\mathfrak{F}_{i}=$ $\left[(0,0),\left(c_{i}^{1}, v_{i}^{c_{i}^{1}}\right), \ldots,\left(c_{i}^{s_{i}}, v_{i}^{c_{i}^{s_{i}}}\right)\right]$, where the first value of each tuple is the number of samples that the node may take and $v_{i}^{c_{i}^{j}}$ is the corresponding information content for those $c_{i}^{j}$ samples. Now, given the fact that more samples will generally generate visual data with a higher information content, we choose a simple linear information valuation function given by $v_{i}^{c_{i}^{j}}=$ $\alpha_{i} \cdot c_{i}^{j}$, where $\alpha_{i}$ is a weighting factor (with support $[0,1])$ that models the typical situation that the sensors within the network are heterogeneous, having different capabilities (i.e. the resolution of their cameras, the quality of their optics, or the sophistication of their image processing algorithms) and varying fields of view. Hence, samples from some sensors will contribute more to the total amount of information collected at the base station than others [18]. However, we remark that our algorithms are not restricted to this linear information valuation function and, in some domains, it may be more valid to model the information as a strictly concave function where continuing to increase the sampling rate generates decreasing gains in information content [19]. ${ }^{1}$ We assume that should the node choose to acquire no samples, it will yield zero information value. Furthermore, we assume that the visual data captured by a node needs to be processed at the base station with that of other nodes, and therefore the information content of the data will only be accounted for if it arrives successfully at the base station.

We further assume that each node has an energy budget, $B_{i}$ (also a private value initially known only to the node), such that its total energy consumption can not exceed this budget. We consider three specific kinds of energy consumption for each node in the network; namely the energy required to (i) acquire, $e_{i}^{\mathrm{s}}$, (ii) transmit, $e_{i}^{\mathrm{Tx}}$, and (iii) receive, $e_{i}^{\mathrm{Rx}}$, a single sample. We disregard the energy required for other types of processing since it is negligible in comparison. Now, since the node has to transmit its own data towards the base station, the total energy required for this activity is thus $E_{i}^{\mathrm{S}}=e_{i}^{\mathrm{s}}+e_{i}^{\mathrm{Tx}}$ per sample (we will later on refer to the combination of these processes as sensing). Similarly, the node could potentially spend a portion of its energy to help its neighbourhood nodes to forward their own samples (and/or data that these nodes are forwarding for another node). This incoming data forwarding process requires a total energy of $E_{i}^{\mathrm{F}}=$ $e_{i}^{\mathrm{Rx}}+e_{i}^{\mathrm{Tx}}$ per sample.

Each node initially stores its collected samples into its local memory buffer in order to be transmitted at a later stage. The transmission period and interval are predetermined. During each transmission phase, the transmitter module of each node is turned on for the purpose of transmitting data or message packets to the base station in a multi-hop fashion since some nodes might be out of the base station's wireless range (see Fig. 2 and 3) and, hence, require the help of others to relay their packets. Battery-powered visual sensor nodes typically offer reasonably small on-board memory and, hence, at the end of the transmission phase, each node's memory buffer is flushed, reinitialized, and ready to store new sampled data [20].

We describe the route through which the samples, $c_{i}^{j}$, will be transmitted to the base station by the vector $R\left(c_{i}^{j}\right)=\left(r_{i}^{1}, \ldots, r_{i}^{b}\right)$, where $r_{i}^{l} \in I$. The first element of this vector is the origin node that actually takes the samples. Each subsequent element of this vector is

\footnotetext{
${ }^{1}$ More comprehensive information valuation functions in the domain of image processing could have also been used, such as those based on the difference in the scene between frames. However, they would typically be too computationally intensive to be run on nodes with the physical constraints of computational and power resources.
} 
unique and $r_{i}^{l}$ will forward the data to $r_{i}^{l+1}$. Thus, for the data value of $c_{i}^{j}$ samples to be taken into account, its routing set must contain the base station node as its last node.

Given the formal description of the problem above, we now wish to maximise the value of the collected data that arrives at the base station. That is, we wish to solve:

$$
\mathcal{D}_{i}^{*}=\arg \max _{\left\{\mathcal{D}_{i}, \mathfrak{F}_{\mathfrak{i}}\right\}} \sum_{i=1}^{n} \sum_{c_{i}^{j} \in C_{i}} d_{i}^{R\left(c_{i}^{j}\right)} v_{i}^{c_{i}^{j}}
$$

In this expression, $d_{i}^{R\left(c_{i}^{j}\right)} \in \mathcal{D}_{i} \in\{0,1\}$ is a decision variable where "1" represents a state where the node carries out the corresponding $c_{i}^{j}$ sampling action and the samples follow the $R\left(c_{i}^{j}\right)$ route to arrive at the base station, and " 0 " represents the state where the node does not carry out the corresponding $c_{i}^{j}$ sampling action. This objective function is maximised subject to the energy budget constraint on each node $i \in I$, such that:

$$
B_{i} \geq c_{i}^{j} E_{i}^{\mathrm{S}}+f_{i} E_{i}^{\mathrm{F}}
$$

where $f_{i}$ represents the total incoming data (or forwarded number samples from its set of neighbourhood nodes $D_{i}$ ), and is given by:

$$
f_{i}=\sum_{d \in D_{i}} c_{d}^{j}+f_{d}
$$

where $i \in R\left(c_{d}^{j}\right)$. Note that the total outgoing number of samples from node $i$ is thus $c_{i}^{j}+f_{i}$. We must also constrain the node to chose one and only one sampling rate, such that:

$$
\sum_{c_{i}^{j} \in C_{i}} d_{i}^{R\left(c_{i}^{j}\right)}=1
$$

for all different possible routes in the network.

Now, consider a simple scenario expected in Fig. 2. Here, nodes $i, 1$, and 3 have their own sets of sampling actions with $s_{i}, s_{1}$, and $s_{3}$ being different numbers of sampling actions respectively. The nodes also have their corresponding energy budgets, $B_{i}, B_{1}$, and $B_{3}$ to perform either sampling and transmitting their own data (requiring $E_{i}^{\mathrm{S}}$ amount of energy per sample) and/or receiving and forwarding the other nodes' data (requiring $E_{i}^{\mathrm{F}}$ amount of energy per sample). For the sake of simplicity, we here assume $E_{i}^{\mathrm{S}}$ requires 8 units of energy and $E_{i}^{\mathrm{F}}$ requires 12 units of energy ${ }^{2}, \forall i \in I$. Each sampling action has a corresponding information value if the samples (collected by the corresponding sampling action) successfully arrive at the base station. In this case, it turns out that the optimal resource allocation for maximising the gathered information value is where node 1 spends its energy to acquire

\footnotetext{
${ }^{2}$ This is just for illustration purposes and our algorithms will naturally work for any values.
}

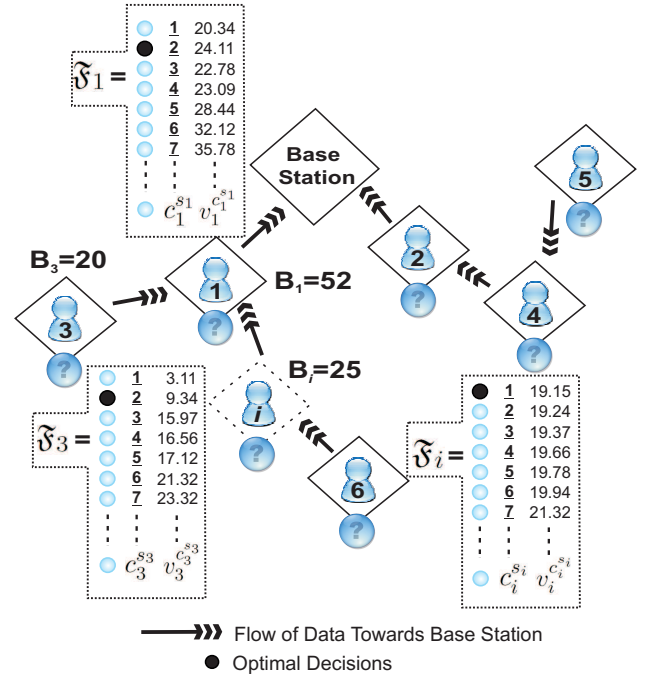

FIGURE 2. A WVSN configured to form a connected and undirected tree structured network (of which any two nodes are connected by exactly one path). Each node only has a single transmission level. We further assume that communication is bi-directional and multiple nodes within range can thus establish a connection. Dotted node $i$ could represent any subtrees in the network.

only two samples, while reserving a portion to relay one and two of nodes $i$ 's and 3's samples respectively. The information value collected at the base station is optimized with these sensing and forwarding actions (acquiring the total value of 52.60).

In the case of a network with loops, as depicted in Fig. 3, node $i$ now has two options to route its data through; namely (i) node 1 which results in route $R\left(c_{i}^{j}\right)=(i, 1$, base station) and (ii) node 2 which results in route $R\left(c_{i}^{j}\right)=(i, 2$, base station). In this case, we simply disregard both nodes 4's and 5's sets of possible sampling actions for the sake of simplicity. With our effective and efficient coordination mechanism (which we will describe shortly in the next section), node 2 will decide to use up all its energy to sample five times as both nodes 4 and 5 do not sample. Node 1 , however, will sample only twice and reserve some of its energy to relay one of each node $i$ 's, 3's and 6's samples. The optimal information value collected with these nodes' behaviour is thus 88.74 .

For both these small sized networks with complete information, a naïve approach to finding the optimal subset of actions is to simply enumerate all possible combinations. This approach, however, is too computationally intensive and works only for very small problems as it very rapidly becomes intractable. For instance, consider the case in which each node, in a tree-structured network, has 30 different sampling actions, the naïve algorithm would now need to evaluate approximately $3 \cdot 10^{29}\left(\prod_{i=1}^{n} c_{i}^{s_{i}}\right)$ solutions (where $n$ is the number of nodes within the network), and considerably more possibilities $\left(\prod_{i=1}^{n}\left(q_{i} \cdot c_{i}^{s_{i}}\right)\right.$, where $q_{i}$ is the total number of unique routes from node $i$ to the 


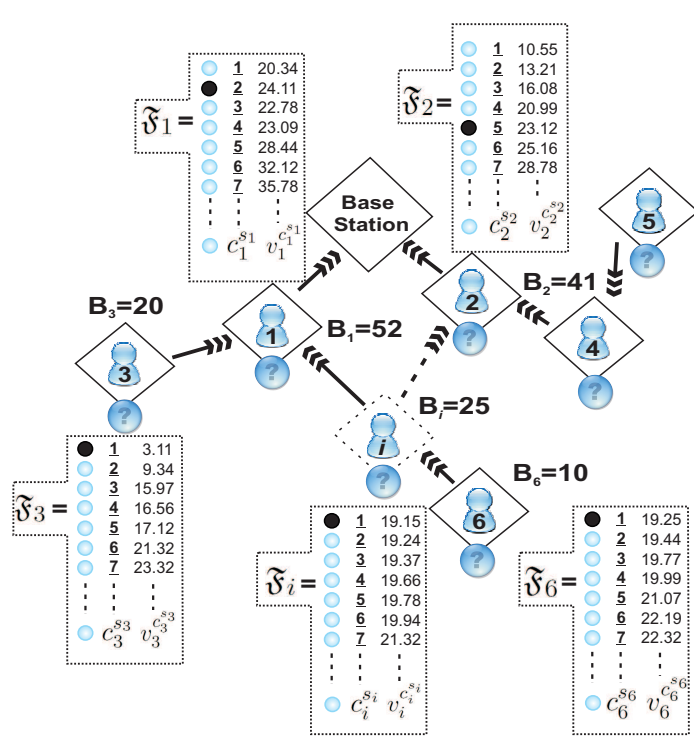

FIGURE 3. A WVSN configured to form an arbitrary network with loops. The dotted arrow represents an alternative flow of data towards the base station.

base station) for the case in a loopy network. These are clearly impossible to compute in a reasonable amount of time regardless of processor speed.

Against this background, the problem as formulated above, is similar to a multiple-choice knapsack problem ${ }^{3}$ (i.e. an NP-complete resource allocation problem) [21], that exhibits the overlapping subproblems ${ }^{4}$ and optimal substructure ${ }^{5}$ properties. Given this insight, we propose algorithms based on the sort of computationally efficient dynamic programming technique that are often used on such knapsack problems for solving this multi-agent distributed coordination problem.

\section{THE ALGORITHMS}

We now focus on the algorithms used by the nodes to make optimal use of their energy resources in order to cooperatively sense, forward, and route data to the base station. Our approach places higher priority on those samples that have a higher information content, and this is achieved by exchanging coordination messages between connected nodes. To this end, we distinguish three types of messages being exchanged by the nodes; namely (i) actual data messages containing visual data sampled by the nodes,

\footnotetext{
${ }^{3}$ There are $m$ items and the set of all items $T=\{1, \ldots, m\}$. Each item $t \in T$ has a value $v_{t}$ and a weight $w_{t}$. The items are subdivided into $o$ categories and exactly one item must be taken from each category. The maximum weight that can be carried in a bag is $G$. Given these, we need to determine which items to include in the bag such that the total weight does not exceed its given limit, while the total value is maximised.

${ }^{4} \mathrm{~A}$ problem with the overlapping subproblems property means that it can be broken down into subproblems which are reused several times.

${ }^{5}$ A problem with the optimal substructure property means that the optimal solution to the problem can be constructed efficiently from optimal solutions to its subproblems.
}

and two types of coordination messages composed of (ii) meta-data messages describing the information content of the visual data together with the number of samples taken to produce that data (i.e. $v_{i}^{c_{i}^{j}}$ and $c_{i}^{j}$ respectively), and (iii) control messages containing the allocation decisions. In WVSNs, the size of the actual data messages overwhelms that of the coordinations messages and, hence, exchanging these before sending the actual data can significantly increase the information collected at the base station by making more efficient use of each node's constrained energy.

The goal of the algorithms that we derive is to calculate the optimal sensing, forwarding, and next-hop decisions of each node. This is given by:

$$
\begin{aligned}
C \max _{I}= & \left\{\left(i, c_{i}^{j}, R\left(c_{i}^{j}\right)\right) \mid d_{i}^{R\left(c_{i}^{j}\right)}=1,\right. \\
& \left.\forall i \in I, \forall c_{i}^{j} \in C_{i}, \forall d_{i}^{R\left(c_{i}^{j}\right)} \in \mathcal{D}_{i}^{*}\right\}
\end{aligned}
$$

and represents a set of 3-tuples indicating for each node in the network, the sensing and forwarding rates that it should adopt, and the route that it should use to transmit its own and its forwarded data to the base station, in order to maximise the objective function in (1), subject to the constraints in (2) and (4). We now present our two novel algorithms. Both of them are efficient as they satisfy the data flow conservation of the network where no energy is wasted by transmitting data that later will not be forwarded to the final destination. We start with the algorithm that assumes that the route by which data is forwarded to the base station is fixed, and then progress to the other that assumes flexible routing.

\subsection{The Algorithm With Fixed Routing}

In this case, each node $i \in I$ can only forward its data to exactly one other node (which will later be referred as its parent). This may be because the underlying communication network of the WVSN is tree structured, or because it actually exhibits loops but an arbitrary choice of route has been made (effectively turning the loopy communication network into a tree). An example of a WVSN whose underlying network structure is a tree structure is shown in Fig. 4. Note that in such tree-structured networks, there is only one unique route between each node and the base station (e.g. $R\left(c_{4}^{j}\right)=\left(4,2\right.$, base station), $R\left(c_{3}^{j}\right)=(3,1$, base station), and $R\left(c_{6}^{j}\right)=(6, i, 1$, base station $\left.)\right)$.

In general, the nodes within a network will deplete their energy resources at different rates since they will have different sampling rates, and will be transmitting and forwarding different quantities of visual data. Each node $i \in I$ thus needs to compute the highest information value it can transmit by using at most $b_{i}^{k} \leq B_{i}$ of its energy. As described earlier, the energy consumption of node $i$ only includes $E_{i}^{\mathrm{S}}$ and $E_{i}^{\mathrm{F}}$ (i.e. the energy to sense and forward a sample respectively). 


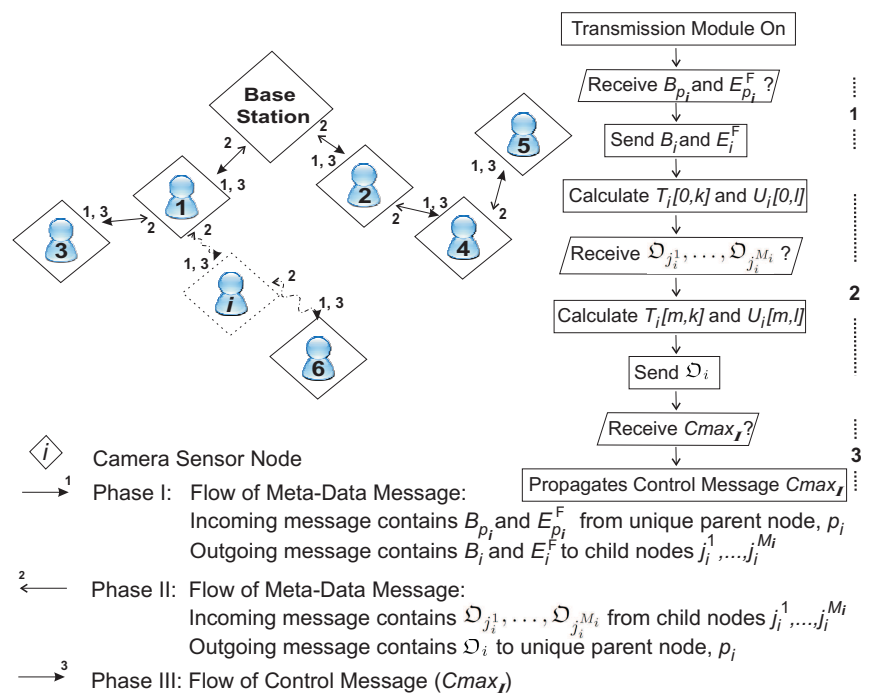

FIGURE 4. The flow of the algorithm in a connected and undirected tree-structured WVSN.

It is therefore sufficient that $b_{i}^{k}$ satisfies:

$$
\begin{aligned}
b_{i}^{k} & =c_{i}^{j} E_{i}^{\mathrm{S}}+f_{i} E_{i}^{\mathrm{F}} \quad \text { where } c_{i}^{j}, f_{i} \geq 0 \\
b_{i}^{k} & \leq B_{i}
\end{aligned}
$$

where $c_{i}^{j}$ is its own number of samples and $f_{i}$ is the number of forwarded incoming samples.

Now, let $\mathfrak{O}_{i}=\left[\left(b_{i}^{1}, \operatorname{Vmax}_{i}^{1}, \operatorname{Cmax}_{i}^{1}\right), \ldots,\left(b_{i}^{K_{i}}\right.\right.$, $\left.\left.\operatorname{Vmax}_{i}^{K_{i}}, C \max _{i}^{K_{i}}\right)\right]$ denote an array of 3 -tuples sorted incrementally by $b_{i}^{k}$ where $k=1, \ldots, K_{i}$, and $b_{i}^{k}$ is the energy limit that satisfies (6) (i.e. $K_{i}$ is the number of feasible $b_{i}^{k}$-s for node $i$ ) which will later on be referred to as the labels of $\mathfrak{O}_{i}$. Vmax $x_{i}^{k}$ is the maximum information value that node $i$ can transmit to its parent by using at most $b_{i}^{k}$, and $C \max _{i}^{k}$ is the set of sensing and forwarding actions that will produce data with the value of $\operatorname{Vmax}_{i}^{k}$. This set of actions is calculated by taking into account its own and its descendants' data.

The algorithm installed on each node runs in three phases (see the state diagram in Fig. 4 and Algorithm 1). In the first, meta-data message propagation is initiated by the base station. To this end, messages containing the value of each node's energy budget, $B_{i}$, and energy consumption for forwarding, $E_{i}^{\mathrm{F}}$, are propagated down the tree (i.e. as soon as any node receives this information from its unique parent node, $p_{i}$ (see state 1 or line 7 ), it sends its own information to its set of children, $J_{i}=\left\{j_{i}^{1}, \ldots, j_{i}^{M_{i}}\right\}$ (line 9)). Having sent this information each node $i$ then enters an idle mode in which it waits for the $\mathfrak{O}$ meta-data arrays from its child nodes. With regard to Fig. 4, the leaf nodes $(3,5$, and 6$)$ eventually receive the meta-data message of nodes 1,4 , and $i$ respectively.

In the second phase, after all the $\mathfrak{O}$ meta-data arrays have arrived from its children (denoted by $\mathfrak{O}_{j_{i}^{1}}, \ldots, \mathfrak{O}_{j_{i} M_{i}}$, see state 2 or lines $\left.14-15\right)$, node $i$ then calculates its own $\mathfrak{O}_{i}$ (line 18). To do so, it constructs a table, $T_{i}$, which has $M_{i}+1$ rows numbered from 0 to $M_{i}$, and $K_{i}$ columns. See Table 1 in which each column $k$ has label $b_{i}^{k}$. Let $T_{i}[x, y]$ denote the element of the table that is in the $x^{\text {th }}$ row and the column with label $b_{i}^{y}$. As every node could choose not to sample (yielding zero value), then $\mathfrak{O}_{j_{i}^{m}}[0]=T_{i}[m, 0]=0$ for all $0 \leq m \leq M_{i}$, where $\mathfrak{O}_{j_{i}^{m}}[x]$ is the $x^{\text {th }}$ element of $\mathfrak{O}_{j_{i}^{m}}$, which is a 3tuple. Moreover, we can assume that the set of labels in each $\mathfrak{O}_{j^{m}}$ that node $i$ has received is the same as the set of labels in its table $T_{i}$. We will show how we can guarantee this later on. $T_{i}$ 's other elements are filled as follows:

$$
\begin{gathered}
T_{i}[0, k]=\max \left\{v_{i}^{c_{i}^{j}}\right\} \\
T_{i}[m, k]=\max _{0 \leq r \leq k}\left\{T_{i}[m-1, r]+\operatorname{Vmax}_{j_{i}^{m}}^{k-r}\right\}
\end{gathered}
$$

for all $1 \leq k \leq K_{i}$ and $1 \leq m \leq M_{i}$, where $\left(c_{i}^{j}, v_{i}^{c_{i}^{j}}\right) \in$ $\mathfrak{F}_{i}$, and $\mathfrak{F}_{i}$ is the array of 2 -tuples defined in the previous section.

According to (7), we can see that $T_{i}[0, k]$ stores the maximum information value of data that can be delivered to node $i$ 's parent by sensing only (with the energy consumption not exceeding the energy limit $b_{i}^{k}$ ). Due to the fact that each of the sets of labels in $\mathfrak{O}_{j_{i}^{m}}$ is equivalent to the set of labels of table $T_{i},(8)$ gives the maximum value of data that node $i$ can deliver to its parent (noting that this data does not only include its own sensed data but also its children's data that will potentially be forwarded through it). Hence, $T_{i}[1, k]$ is the maximum value that can be sent by taking into account the sensed data and the data from $j_{i}^{1}$, with respect to the $b_{i}^{k}$ energy limit. $T_{i}[2, k]$ stores the maximum value when the data from child node $j_{i}^{2}$ is also included. In general, $T_{i}[m, k]$ is the maximum information value that node $i$ can transmit to its parent, given the $b_{i}^{k}$ energy limit. The data considered is the potential forwarded data from child nodes $j_{i}^{1}, \ldots, j_{i}^{m}$ and node $i$ 's own sensed data.

Note that while it is necessary to construct the entire table, as in conventional dynamic programming solutions to the multiple-choice knapsack problem, it is only the last row that provides useful meta-data regarding the maximum information value of data that can be transmitted given different feasible values of $b_{i}^{k}$. Indeed, it is only the last element of this row that represents the maximal information value that node $i$ can transmit to the parent node.

To illustrate how the elements of the table are calculated in a clearer way, consider Tables 1 and 2 in which the information values of node $i$ 's sensed data and the $V \max _{j_{i}^{m}}^{k}$ values of $\mathfrak{O}_{j_{i}^{m}}$ arriving from its child nodes $j_{i}^{m}$ respectively are chosen arbitrarily for illustrative purposes. The rows of Table 1 represent the set of nodes whose data has been taken into account. For instance where row $=i$, if node $i$ has $b_{i}^{0}, b_{i}^{1}, b_{i}^{2}, b_{i}^{3}$, or $b_{i}^{4}$ amount of energy limit, in return it will be able to 


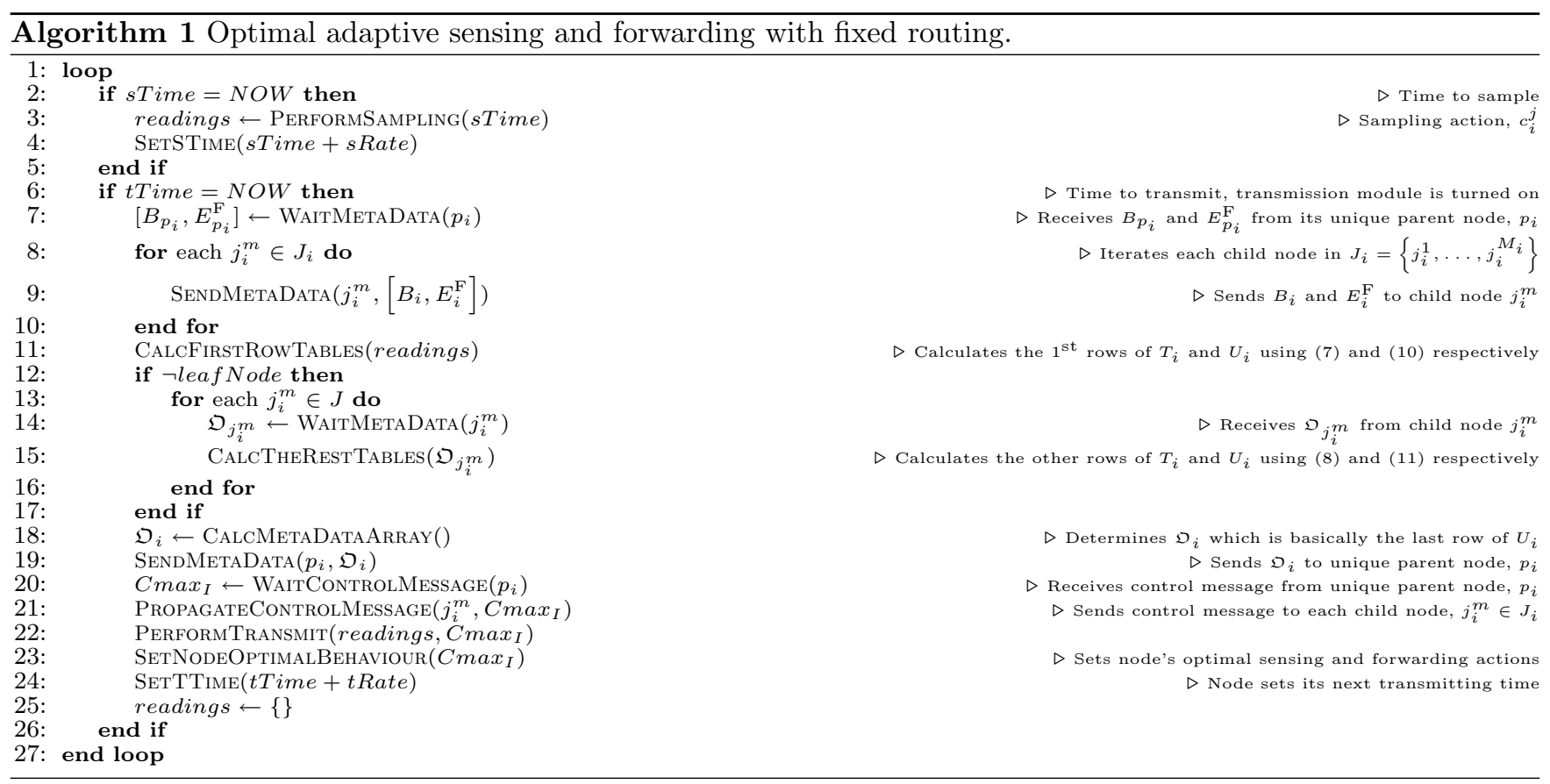

sense it own data with the maximum value of $0,12.34$, $14.56,28.25$, or 50.98 correspondingly. These values are calculated using $(7) . \quad \mathfrak{O}_{j_{i}^{1}}$ then arrives (see Table 2 where row $\left.=\mathfrak{O}_{j_{i}^{1}}\right)$ from its child node $j_{i}^{1}$ containing the maximum values that this node could potentially forward to node $i$.

The elements of $T_{i}$ 's second row (i.e. row $=\left\{i \cup j_{i}^{1}\right\}$ ) can thus be calculated using (8). These elements represent the maximum information that node $i$ could send by taking into account not only its own sensed data, but also the data that could be potentially forwarded from its child node $j_{i}^{1}$. For instance where column $=b_{i}^{1}$, node $i$ could allocate all its $b_{i}^{1}$ energy resources to either sense and transmit its own data or to forward data from its child node $j_{i}^{1}$. In this case, the node chooses to sense and transmit its own data since it has a higher value. Where column $=b_{i}^{2}$, however, the node could again allocate all its $b_{i}^{2}$ energy resources to either sense its own data or to forward its child node $j_{i}^{1}$ 's data. Alternatively it could as well divide its $b_{i}^{2}$ energy resources by allocating a portion of $b_{i}^{1}$ energy resources to its own and another $b_{i}^{1}$ to its child node. In this case, it turns out that the latter alternative yields the highest information value of 19.32. $T_{i}$ 's other elements are calculated in a similar way.

Now, the next step of the algorithm is to calculate $\mathfrak{O}_{i}$. To do so, let $U_{i}$ denote a table similar to $T_{i}$. However, its labels $b_{i}^{l}$, now, satisfy the following:

$$
\begin{aligned}
& b_{i}^{l}=\left(c_{i}^{j}+f_{i}\right) E_{p_{i}}^{\mathrm{F}} \quad \text { where } c_{i}^{j}, f_{i} \geq 0 \\
& b_{i}^{l} \leq B_{p_{i}}
\end{aligned}
$$

where $B_{p_{i}}$ is the energy budget of $i$ 's unique parent node, $p_{i}$, and $E_{p_{i}}^{\mathrm{F}}$ is the value of energy consumption of the parent for forwarding a sample. Recall that these values were delivered to node $i$ in the first stage. Let $L_{i}$ denote the number of all $b_{i}^{l}$ that satisfy (9). Similarly, we can calculate table $U_{i}$ 's elements in a similar fashion to those of $T_{i}$ as described earlier, but with the new labels:

$$
U_{i}[0, l]=\min \left(\max \left\{v_{i}^{c_{i}^{j}}\right\}, T_{i}\left[0, K_{i}\right]\right)
$$

$U_{i}[m, l]=\min \left(\max _{0 \leq r \leq l}\left\{U_{i}[m-1, r]+\operatorname{Vmax}_{j_{i}^{m}}^{l-r}\right\}, T_{i}\left[m, K_{i}\right]\right)$

for all $1 \leq l \leq L_{i}$ and $1 \leq m \leq M_{i}$, where $\left(c_{i}^{j}, v_{i}^{c_{i}^{j}}\right) \in \mathfrak{F}_{i}$.

We can now construct the meta-data array of node $i$ such that $\mathfrak{O}_{i}=\left[\left(b_{i}^{1}, U_{i}\left[M_{i}, 1\right], C \max _{i}^{1}\right), \ldots\right.$, $\left.\left(b_{i}^{L_{i}}, U_{i}\left[M_{i}, L_{i}\right], C \max _{i}^{L_{i}}\right)\right]$, where $U_{i}\left[M_{i}, l\right]$ is the maximum information value that node $i$ can transmit to its parent node (by using at most $b_{i}^{k}$ energy) which can subsequently forward the received $i$ 's data by using at most $b_{i}^{l}$ energy. $C \max _{i}^{l}$ is the set of sensing and forwarding actions that will produce data with the value of $U_{i}\left[M_{i}, l\right]$. Hence, once $\mathfrak{O}_{i}$ is sent to the parent node, its labels will be the same as those in table $T_{p_{i}}$ of the parent node. This second phase meta-data message containing $\mathfrak{O}_{i}$ propagates up the network arriving back at the base station (line 19).

In the third phase of the algorithm, each parent node will have received meta-data arrays from all of its children. The base station will be able to calculate the highest information value it can potentially receive from all the nodes beneath it in the network. Based on the structure of $\mathfrak{O}_{i}$, each node $i$ can easily determine what amount of data it should receive from each child node and, hence, how many samples it should acquire and transmit itself. A control message containing this set is then propagated down the network (see state 3 or 
TABLE 1. The $T_{i}$ table of node $i$. Its $\mathfrak{O}_{i}$ meta-data array is represented by the dotted rectangle.

\begin{tabular}{|r|c|c|c|c|c|c|}
\hline $\boldsymbol{T}_{i}$ & $\boldsymbol{b}_{i}{ }^{0}$ & $\boldsymbol{b}_{i}{ }^{1}$ & $\boldsymbol{b}_{i}{ }^{2}$ & $\boldsymbol{b}_{i}{ }^{3}$ & $\boldsymbol{b}_{i}{ }^{4}$ & $\boldsymbol{b}_{i}{ }^{k}$ \\
\hline$\{\boldsymbol{i}\}$ & 0 & 12.34 & 14.56 & 28.95 & 50.98 & $\vdots$ \\
$\left\{\boldsymbol{i} \cup \boldsymbol{j}_{i}^{1}\right\}$ & 0 & 12.24 & 19.32 & 45.89 & 58.23 & $\vdots$ \\
\hdashline & $\vdots$ & $\vdots$ & $\vdots$ & $\vdots$ & $\vdots$ & $\vdots$ \\
\hdashline $\left.\boldsymbol{i} \mathbf{U} \boldsymbol{j}_{i}^{1} \mathbf{U} \ldots \mathbf{U}_{i}^{\boldsymbol{j}_{i}}\right\}$ & 0 & 12.34 & 28.78 & 45.89 & 58.23 & $\vdots$ \\
\hline
\end{tabular}

TABLE 2. $\mathfrak{O}_{j_{i}^{m}}$ meta-data arrays that have arrived from each child nodes $j_{i}^{1}, \ldots, j_{i}^{M_{i}}$.

\begin{tabular}{|c|c|c|c|c|c|c|}
\hline $\mathfrak{O}_{j_{i}^{m-\mathrm{s}}}$ & $\boldsymbol{b}_{i}^{0}$ & $\boldsymbol{b}_{i}^{1}$ & $\boldsymbol{b}_{i}^{2}$ & $\boldsymbol{b}_{i}^{3}$ & $\boldsymbol{b}_{i}^{4}$ & $\boldsymbol{b}_{i}^{k}$ \\
\hline $\mathfrak{O}_{j_{i}^{1}}$ & 0 & (6.98) & 15.67 & 45.89 & 48.99 & \\
\hline $\mathfrak{O}_{j_{i} M_{i}}$ & 0 & 6.79 & 28.78 & 35.89 & 51.88 & \\
\hline
\end{tabular}

lines 20-21), and this control message informs each node of its optimal decisions (lines 22-23). In this way, there is a guarantee that all of the data transmitted by each node will reach the base station. The control message eventually reaches the leaf nodes which then start to acquire and transmit visual data as planned.

\subsection{The Algorithm with Flexible Routing}

Next, we consider the algorithm which assumes flexible routing, and makes optimal decisions regarding both the sensing and forwarding actions that each node should perform, and also the route by which data should be forwarded to the base station (see Fig. 5 for an illustration of this case). In order to make the routing decision tractable, we place one minor restriction on the routes that our algorithm can consider. Specifically, we assume that the nodes always forward their data toward the base station; that is, they will not forward data to a node that is further from the base station (in terms of hop count) than themselves. We believe this is a reasonable assumption. There may be cases where several nodes are better off taking longer paths. However, in general such paths will deplete the energy resources of a greater number of nodes, and are thus unlikely to be optimal solutions. Furthermore, we assume that the data samples of a node can only be sent as a bundle (i.e. they are indivisible). The data readings of different nodes can, however, be sent through different routes to the base station if there is more than one option to choose from.

With these assumptions, we now need to organize the nodes into different levels. To this end, a network simulator using a $B G P$-based ${ }^{6}$, robust, and scalable routing discovery TCP/IP protocol is developed. Our coordination mechanism will therefore sit on top of this

\footnotetext{
${ }^{6} \mathrm{BGP}$ stands for Border Gateway Protocol and it is a common network routing protocol on the Internet [22].
}

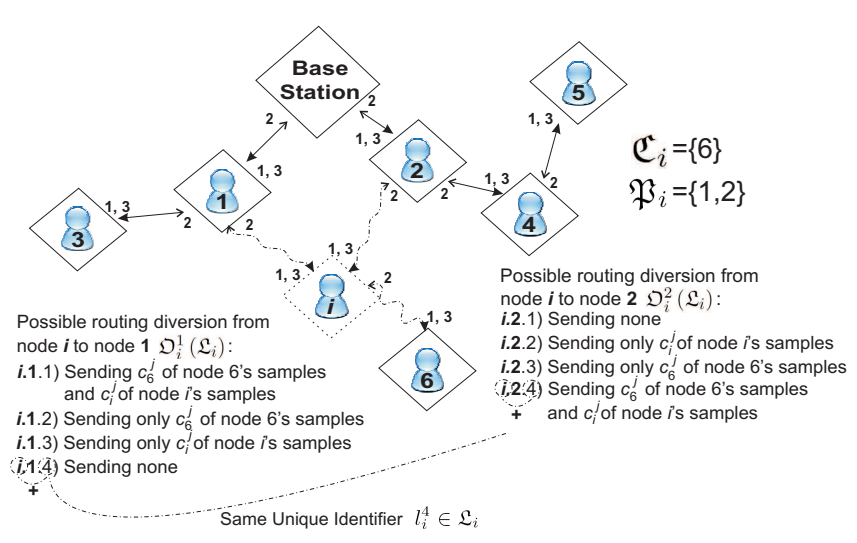

FIGURE 5. The flow of the algorithm that assumes flexible routing and makes optimal decisions regarding both sensing, forwarding, and next-hop (or routing) decisions. The phases involved in this algorithm are similar to those in the algorithm for fixed routing.

network protocol. The first level consists of all the nodes that have a 1-hop shortest path to the base station (nodes 1 and 2 in Fig. 5). Nodes that belong to the second level have a 2-hop shortest path to the base station (nodes $i, 3$, and 4). Nodes 5 and 6 have a 3 hop shortest path. Now, according to this hierarchy, each node can only forward its data to higher level nodes within its transmission range. In Fig. 5, for example, node $i$ has two potential shortest routes to choose from; namely (i) node 1 which results in route $R\left(c_{i}^{j}\right)=(i, 1$, base station) and (ii) node 2 which results in route $R\left(c_{i}^{j}\right)=(i, 2$, base station). Node $i$ does not consider routing through node 6 since 6 is a greater hop count away from the base station than it is.

Furthermore, as we can see from the figure, node $i$ has potentially two bundles of data to consider (its own and data that it is forwarding for node 6 ). In addition, it has two possible shortest paths to choose between (either through node 1 or 2 for each of the bundled data). Thus, a number of routing options exist for this node. It could send both bundles of data through node 1 , such that $R\left(c_{i}^{j}\right)$ contains $(i, 1, \ldots)$ and $R\left(c_{6}^{j}\right)$ contains $(\ldots, i, 1, \ldots)$, or it could send them through node 2 , such that $R\left(c_{i}^{j}\right)$ contains $(i, 2, \ldots)$ and $R\left(c_{6}^{j}\right)$ contains $(\ldots, i, 2, \ldots)$. Other alternatives are to send each of them separately through each possible route, such that $R\left(c_{i}^{j}\right)$ contains $(i, 1, \ldots)$ and $R\left(c_{6}^{j}\right)$ contains $(\ldots, i, 2, \ldots)$, or the other way around.

Now, let $\mathfrak{P}_{i}$ denote the set of parent nodes (which are nodes with a one hop shorter shortest path to the base station) of node $i$ and $\mathfrak{C}_{i}$ denote the set of its descendants. Thus, at each node $i \in I$, there are at most $\left|\mathfrak{P}_{i}\right|^{\left|\mathfrak{C}_{i}\right|+1}$ possibilities to forward the bundled data, where $\left|\mathfrak{P}_{i}\right|$ and $\left|\mathfrak{C}_{i}\right|$ are the sizes of $\mathfrak{P}_{i}$ and $\mathfrak{C}_{i}$ respectively. This is because each node has to forward $\left|\mathfrak{C}_{i}\right|+1$ bundles through $\left|\mathfrak{P}_{i}\right|$ different shortest paths. Next, let $\mathfrak{L}_{i}$ denote the set of these possibilities (with $\left|\mathfrak{L}_{i}\right|=\left|\mathfrak{P}_{i}\right|^{\left|\mathfrak{C}_{i}\right|+1}$ ) and each $l_{i}^{t} \in \mathfrak{L}_{i}$, 


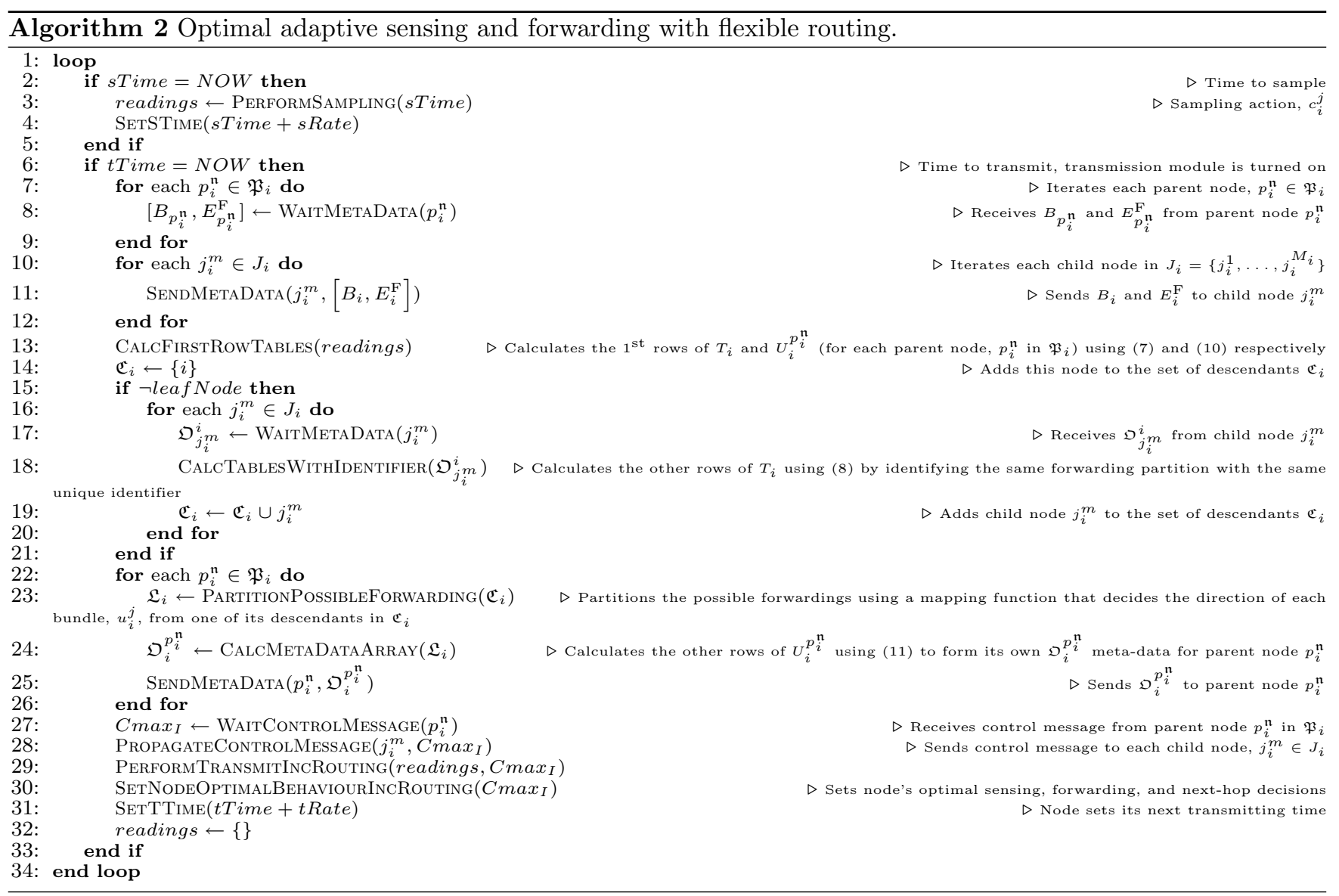

a possible partition of forwarding at node $i$. That is, $l_{i}^{t}=\left[F\left(u_{i}^{1}\right), \ldots, F\left(u_{i}^{\left|\mathfrak{C}_{i}\right|+1}\right)\right]$ where $u_{i}^{j}$ is the $j^{\text {th }}$ bundle that might arrive at node $i$ from one of its descendants, $F\left(u_{i}^{j}\right)$ is a mapping function that decides the forwarding direction (or path) for this particular bundle, and $u_{i}^{\left|\mathfrak{C}_{i}\right|+1}$ is node $i$ 's own bundle of samples.

Given this, our algorithm with flexible routing is similar to that with fixed routing, and as before, it runs in three phases (see Algorithm 2). The first, in which the parent nodes send their information regarding $B_{p_{i}^{\mathfrak{n}}}$ and $E_{p_{i}^{\mathrm{n}}}^{\mathrm{F}}$ to their child nodes (where $p_{i}^{\mathfrak{n}} \in \mathfrak{P}_{i}$ ), is identical (see lines 7-13). There are, however, slight modifications in the next phase. These modifications are needed to keep track of all the possible partitions of forwarding for nodes which have more than one shortest path routes to the base station.

In more detail, in the second phase, instead of sending one $\mathfrak{O}_{i}$ to a unique parent (as in the case of treestructured networks), here, each node $i$ has to calculate all the $\mathfrak{O}_{i}^{p_{i}^{\mathrm{n}}}\left(l_{i}^{t}\right)$ meta-data arrays for each $l_{i}^{t} \in \mathfrak{L}_{i}$ partition of forwarding for each $p_{i}^{\mathfrak{n}} \in \mathfrak{P}_{i}$ (see lines 2325). Specifically, this is achieved by first calculating the $T_{i}$ table as we did for the first algorithm (line 17). In this case, however, we join each of the arriving $\mathfrak{O}_{j_{i}^{m}}^{i}\left(l_{j_{i}^{m}}^{t}\right)$ from its children $j_{i}^{1}, \ldots, j_{i}^{M_{i}}$ with those that belong to the same forwarding partition with the same unique identifier (line 18). The unique identifier is formed and attached to a particular partition of forwarding when there are more than one possible routes to forward to (line 23). As in Fig. 5, a feasible unique identifier could be the index of $l_{j_{i}^{m}}^{t}$. Next, we calculate $U_{i}^{p_{i}^{\mathfrak{n}}}$ tables for each $p_{i}^{\mathfrak{n}} \in \mathfrak{P}_{i}$ as in the first algorithm (line 24).

The rest of the second phase and third phase remain the same as that of the algorithm with fixed routing described previously (see lines 27-30).

\section{EMPIRICAL EVALUATION}

Having described the algorithms with fixed and flexible routing, we now seek to evaluate their performance and effectiveness when applied to typical WVSNs whose communication networks exhibit loops. Our goal in this empirical evaluation is to quantify the performance of the algorithms in terms of the quantity of information that they deliver to the base station, and the communication and computational costs of the coordination. We expect the algorithm with flexible routing to deliver more information, but make greater demands of computation and communication resources (because of the large number of alternative routes for the data that it must evaluate). However, given that the algorithm with fixed routing can always be applied in this setting by ignoring the fact that there 
exist alternative routing options, and just making an arbitrary choice, we are interested in the tradeoff between the loss in information and the saving in resources that results. We first describe the experimental setup and then go on to the actual evaluation.

\subsection{Network and Parameters Setup}

In our experiments, we create instances of a WVSN by randomly deploying the nodes within a unit square (i.e. $x$ and $y$ coordinates within the square are randomly drawn from a uniform distribution with support $[0,1])$, and connecting them according to a randomly determined radio transmission range (extending this range as necessary to ensure that there are no unconnected nodes). Each resulting WVSN exhibits a loopy communication network such that for each node there are multiple alternative routes to the base station.

We consider twenty different sampling actions for each node such that the possible sampling rates, $C_{i}$, of each node are initialized to $C_{i}=\{1, \ldots, 20\}$. The corresponding information content $v_{i}^{c_{i}^{j}}$ for each of the $c_{i}^{j} \in C_{i}$ samples is generated using the generic information metric (defined in Sect. 2), with the factor, $\alpha_{i}$, randomly drawn from a uniform distribution with support $[0,1]$.

The energy budget of each node is randomly generated (drawn from the same distribution) with a predetermined maximum value that ensures the network as a whole is energy constrained, and no node is able to sample and transmit at its maximum rates. We scale this predetermined maximum value with the number of nodes in the network since larger networks require sensors to forward data for a larger number of nodes. We assume that each real valued number inside a coordination message (e.g. the value of node $i$ 's energy budget, $B_{i}$, or its optimal sampling rate, $c_{i}^{j}$ ) occupies 4 bytes of communication cost, and the energy consumption for sensing and forwarding a sample is fixed throughout the entire experiment. ${ }^{7}$

We apply our algorithm with flexible routing just once, directly on the loopy communication network of the WVSN (see Fig. 6(a) for an exemplar scenario), such that it determines both the optimal sensing and forwarding actions, as well as the routes. Prior to applying our algorithm with fixed routing, we allow each node to make an arbitrary choice of the route that its data (and any data that it forwards for other nodes) will take toward the base station. This effectively turns the loopy communication network into a tree-structured one, with each node effectively selecting their parent in

\footnotetext{
${ }^{7}$ Note that we do not consider the failure, addition, or removal of nodes. Also, we do not consider the dropping or corruption of meta-data or control message packets, and hence assume that message packets are always transferred successfully to the destination.
}

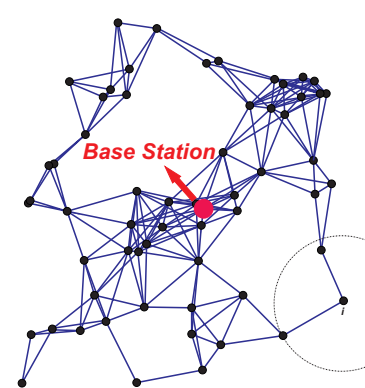

(a)

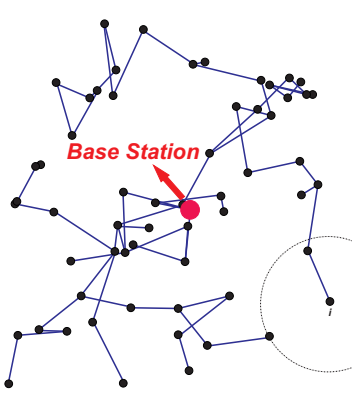

(b)
FIGURE 6. (a) A randomly created and connected WVSN (of 60 nodes) whose underlying communication network exhibits loops. (b) The resulting tree-structured network formed when each node makes an arbitrary choice of the route that its data will take toward the base station. The dotted circle in each graph represents the wireless range of node $i$. Neighbourhood nodes within this transmission range can hear the transmitted messages of the node. In both these networks, all nodes are set with the same transmission range.

the tree (see Fig. 6(b)). We then apply our algorithm with fixed routing to calculate the optimal sensing and forwarding decisions of each node. For each instance of the WVSN, we repeat this process 100 times, averaging over the unique instances of trees that result.

We perform repeated experiments by creating 100 instances of the WVSN with $6,9,12,15, \ldots, 60$ nodes. The algorithm with flexible routing, however, is only run with up to 21 nodes. This is solely due to the insufficient memory allocated for the java heap space that is used to keep track all possible partitions of forwarding which grows exponentially with the number of nodes and potential routes.

\subsection{Benchmark Algorithm}

In this experiment, we also benchmark our two algorithms against a uniform non-adaptive algorithm with fixed routing. This algorithm dictates that each sensor $i \in I$ in the network should simply choose to allocate its energy budget, $B_{i}$, equally to itself and each of its descendants such that it will naïvely sample and transmit the minimum of $\left(\frac{B_{i}}{\left|\mathfrak{C}_{i}\right| \cdot E_{i}^{\mathrm{S}}}, \frac{B_{p_{i}}}{\left|\mathfrak{C}_{p_{i}}\right| \cdot E_{p_{i}}^{\mathrm{S}}}\right)$ times regardless of whether the samples will eventually be forwarded towards the base station. $\left|\mathfrak{C}_{i}\right|$ and $\left|\mathfrak{C}_{p_{i}}\right|$ are the numbers of descendants of node $i$ and node $i$ 's parent, $p_{i}$, respectively, and $B_{p_{i}}$ is the energy budget of node $p_{i} . E_{i}^{\mathrm{S}}$ and $E_{p_{i}}^{\mathrm{S}}$ are the energy required by node $i$ and $p_{i}$ correspondingly in order to sense a sample.

\subsection{Results}

We present the results of the simulation process described above in Fig. 7, 8, and 9. We note that the standard error in the mean is close to the size of the plot symbols and, thus, error bars are omitted from 


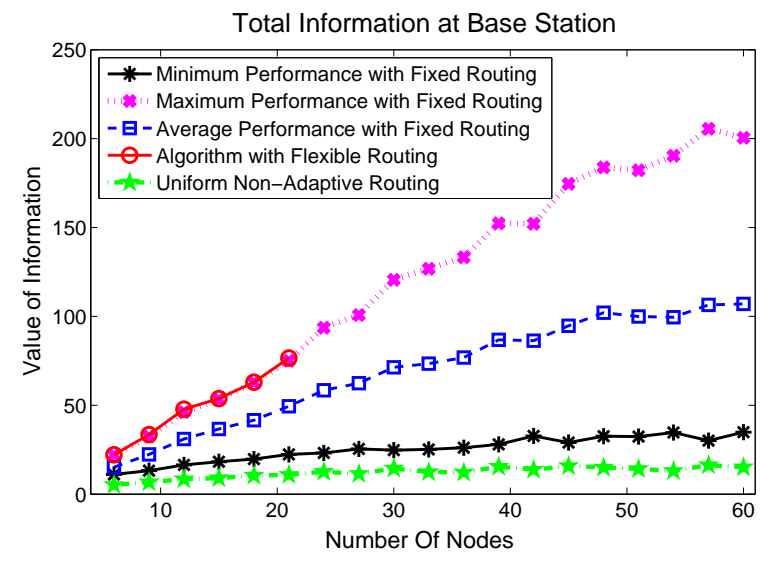

FIGURE 7. Simulation results showing the performance of the algorithms with flexible, fixed (with maximum and minimum performance), and uniform non-adaptive routing against total information collected at the base station.

the plots for clarity.

Considering first Fig. 7, we observe that the algorithm with flexible routing delivers close to twice the quantity of information to the base station compared to the algorithm with an arbitrary fixed routing. This is as expected since in loopy communication networks, there are typically many alternative routes available for routing data, and the flexible algorithm is able to exploit them to deliver the maximum possible information to the base station. Note that the quantity of information delivered does not increase monotonically, but decreases in a number of cases. This effect is due to the way in which we have scaled the maximum energy budget of the nodes as the network increases in size. This scaling fails to fully account for the necessary increase in sample forwarding and, thus, the network becomes increasingly energy constrained as it grows in size. The uniform nonadaptive algorithm, however, performs poorly as it has no intelligence of adapting the nodes' actions.

In the same figure, we also show the mean maximum and minimum performance (that is, for each loopy network, we record the performance of the best and worst tree, and then average over each loopy network that we test) of the algorithm with fixed routing. We remark that by making an appropriate choice of parent, we can derive performance close to that of the algorithm with flexible routing without incurring any additional computation or communication cost as will be explained shortly.

However, the increased information delivered by the algorithm with flexible routing comes at considerable communication and computational cost. Fig. 8 and 9 show the total size of coordination messages exchanged by the nodes and the average computation time of each node. Note that these figures are presented on a logarithmic scale for a clearer visualization. Specifically,

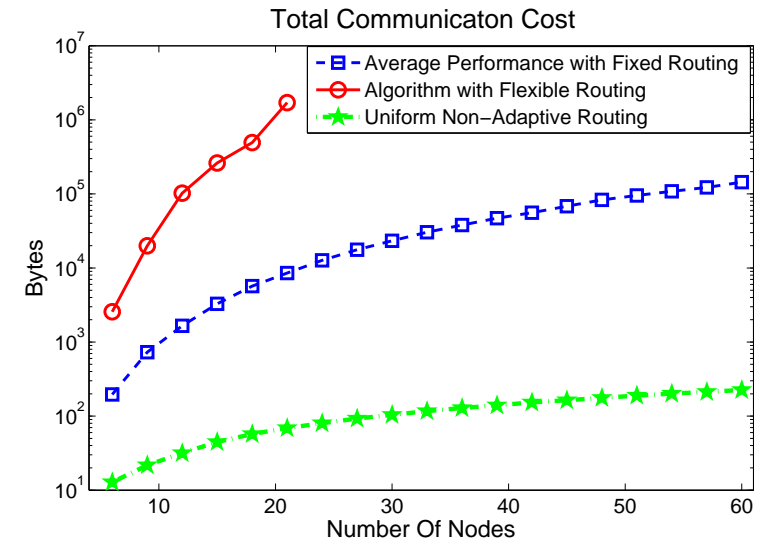

FIGURE 8. Simulation results showing the performance of the algorithms with flexible, fixed (with maximum and minimum performance), and uniform non-adaptive routing against total communication cost for coordination.

Fig. 8 shows that typically only a few tens of kilobytes of coordination message packets are required by the algorithm with fixed routing, while the algorithm with flexible routing exhibits approximately two orders of magnitude more; with a few megabytes of coordination message packets being exchanged. The increase is due to the way in which the flexible routing algorithm keeps track all possible partitions of forwarding that grow exponentially with the number of nodes and potential routes.

Likewise, Fig. 9 shows that the average computation time of a node required by the algorithm with fixed routing is typically less than 1 millisecond, while that of the algorithm with flexible routing approaches 100 milliseconds (a two orders of magnitude increase). ${ }^{8}$ The increase in terms of computation time is due to the additional time which the flexible routing algorithm requires in order to enumerate each possible partition of forwarding.

Speaking more generally, these results indicate that the algorithm with flexible routing is able to deliver significantly more information to the base station, but it incurs a considerable additional computation and communication cost in doing so. The choice of algorithm thus largely depends on the actual application domain. If the network is small, and the size of the actual data messages is large, then the algorithm with flexible routing is most appropriate. However, this algorithm scales poorly as the size or connectivity of the network increases (due to the exponential growth in the number of possible combinations of routing options that it must evaluate). In such cases, the size of the coordination messages may rapidly approach that of

\footnotetext{
${ }^{8}$ These measurements were performed on a $3 \mathrm{GHz}$ desktop PC. Typically, the nodes within a WVSN will use much lower powered processors and, thus, while we would expect the ratio between the algorithms to be the same, the overall computation time is likely to be longer.
} 


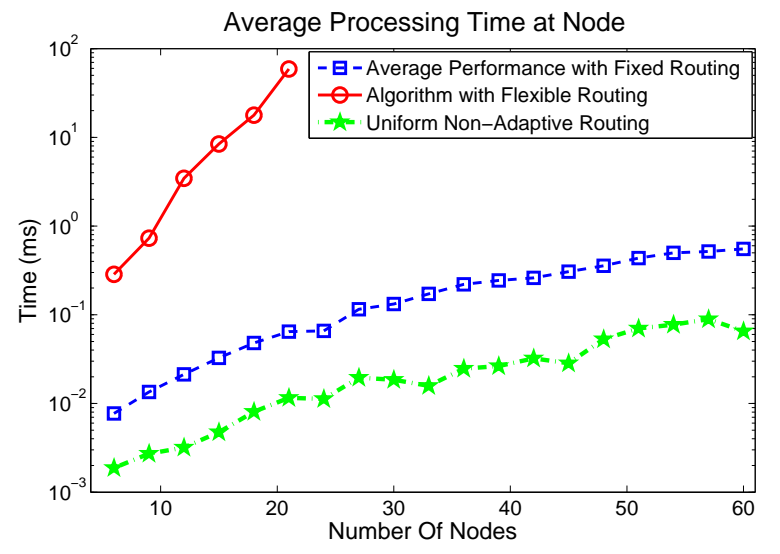

FIGURE 9. Simulation results showing the performance of the algorithms with flexible, fixed (with maximum and minimum performance), and uniform non-adaptive routing against average computation time at each node.

the actual data messages, and hence, coordination may not actually yield any energy saving. To address this, the algorithm with fixed routing may be run on the original loopy network by ignoring the fact that there exist alternative routing options, and having each node make an arbitrary choice of route. While the quantity of information delivered to the base station will be reduced (by up to $50 \%$ in our experiments), this solution will scale well and use minimal communication and computational resources.

\section{RELATED WORK}

The work that is most closely related to ours is that of Padhy et al. who develop a decentralised adaptive sampling and routing protocol [23]. Within this mechanism, each node adjusts its sampling rate depending on a valuation function that assigns a value to newly sampled data. This protocol is intended for low power, computationally constrained devices, and as such, relies on a heuristic approach to estimate the opportunity energy cost used by each sensor for sensing, forwarding, and routing data. The protocol is not efficient and the integration of the node's actions is very limited since there is no guarantee that the transmitted data will actually be forwarded to the base station. For instance, there might be cases where nodes with data of a high value are unable to send their data to the base station because intermediate nodes have depleted their energy. The protocol could thus result in no data collection.

In a somewhat similar setting, but still concerned with the decentralised approaches to decision making within sensor networks, Mainland et al. present a market-based approach for determining efficient node resource allocations [24]. Rather than manually tuning node resource usage, or providing specific algorithms as we do here, their approach defines a virtual market in which nodes sell goods (e.g. data sampling, listening, or forwarding) in response to global price information that is established by the end user. However, this approach involves an external coordinator to set prices in order to induce any particular global behaviour, and it is not clear how this price determination should be performed in order to elicit desirable system-wide properties.

Within agent-based approaches, a useful technique that has emerged for solving multi-agent distributed coordination problems is that of distributed constraint optimization. To date, there has been a rich set of real-world distributed applications, such as in the domain of networked systems, for which this technique has been used, particularly, in distributed applications where constraints exist among agents. In more detail, a number of algorithms in this area have been developed; these include Synchronous Branch and Bound (SBB) [25], Distributed Breakout Algorithm (DBA) [26], Asynchronous Distributed Optimization (ADOPT) [27], Distributed Pseudotree Optimization Procedure (DPOP) [28], Asynchronous Partial Overlay (APO) [29], and Max-Sum [30]. Now, many of these algorithms are also based on the dynamic programming method because it is often used to solve problems with distinguished properties of overlapping subproblems and optimal substructure. The method works by (i) breaking down the multi-agent problem into smaller subproblems, (ii) solving these subproblems optimally using this three-step process recursively, and (iii) constructing an optimal solution to the original problem by using these optimal solutions of the subproblems.

However, SBB is found to be ineffective due to its slow performance. Moreover, it uses the notion of a virtual agent that acts as a central manager to deliver messages among agents and that is aware of all of the delivered messages' state. DBA is preferable when we want a near optimal solution much quicker than SBB, however, it may fail to return a solution even if one exists and also it cannot correctly determine if no solution exists.

To counter the limitations introduced by systematic, synchronous, and sequential message exchanges between nodes, ADOPT and DPOP are guaranteed to converge to the optimal solution while using only localized asynchronous communication and computation in the setting of a sensor resource allocation problem. However, since these algorithms are complete and asynchronous, they require an exponential increase in either the total message size being exchanged or the memory complexity on the agents (unlike the case of our algorithm with fixed routing). This is unrealistic for sensor networks in which the nodes are typically installed with limited computational, storage, and memory resources. Moreover, they are not specifically tailored to the specific problem that we address here. We, however, adopt a similar message propagation method where each node coordinates and exchanges meta-data regarding the utility of the actual data with its neighbourhood nodes before sending the actual data itself. 
This is done in order to efficiently make use of the limited energy resources.

In contrast, APO uses centralised mediator agents to compute the solution for portions of the original problem and, therefore, lacks distributed control. Finally, Max-Sum exchanges messages between conflicting lowpower sensor nodes using a cyclic bipartite factor graph representation. It scales very well in tree-structured graphs and generates approximate solutions close to the global ones returned by ADOPT and DPOP. However, it is not a complete algorithm, and may fail to converge in cyclic graphs as there is no built-in termination algorithm to stop the message exchange process.

In a different setting, Pizzocaro et al. try to optimize a sensor mission assignment problem by modelling it as a NP-complete multiple knapsack problem [31]. They introduce the sensor utility maximization model in which each sensor-mission pair is associated with a utility offer. Each mission might require and compete for the same sensors, however, a sensor can only be assigned to serve only one mission at any point of time. A profit function is formulated and this represents the value that a sensor can bring to a mission. The goal is therefore to maximise the total profit, while ensuring that the total utility cumulated by each mission does not exceed its uncertain demand (otherwise, some utility offer will be wasted). A novel greedy algorithm is developed and showed to perform better compared to a number of benchmarks by offering the best trade-off between the quality of the returned solution and the computational cost. However, the algorithm considered here follows a centralised approach, where all the intensive computation takes place at a central node which has all the information about the system's state. The dynamism of the sensor-mission pair, where a mission may change or adapt its priority value over time if its demand is not met after a certain period of time, is also missing in this work.

\section{CONCLUSIONS}

In this article, we have considered the problem of inter-related adaptive sensing, forwarding, and routing within WVSNs in order to manage the limited energy resources of nodes in an effective and efficient way. We have developed two novel optimal decentralised algorithms: one which assumes fixed routing and calculates the optimal sensing and forwarding actions that each node should perform, and one which assumes flexible routing, and makes optimal decisions regarding both the integration of actions that each node should choose, and also the route by which data should be forwarded to the base station. In an empirical evaluation, we showed that the algorithm with flexible routing delivered approximately twice the quantity of information to the base station, but at a considerably higher communication and computational cost. Thus, while the algorithm with flexible routing is suitable for networks with a small numbers of nodes, it scales poorly, and as the size of the network increases, the algorithm with fixed routing is favoured.

Our ongoing work in this area includes relaxing the restriction that the nodes may only forward data to nodes that are closer to the base station (in terms of hop count) than themselves and, in particular, we would like to characterise the circumstances in which this may yield some benefit. More significantly, we would also like to develop a principled algorithm for making the choice of route when applying the algorithm with fixed routing to loopy WVSNs (rather than having the nodes make an arbitrary choice of parent in order to convert the loopy network into a tree-structured network as we have done here). Our empirical results indicate that the performance of the algorithm with fixed routing is very close to that of the algorithm with flexible routing if the appropriate fixed route is selected (see Fig. 7), ${ }^{9}$ and thus, there is great potential in doing so.

\section{REFERENCES}

[1] Cardell-Oliver, R., Smettem, K., Kranz, M., and Mayer, K. (2005) A reactive soil moisture sensor network: Design and field evaluation. International Journal of Distributed Sensor Networks, 1, 149-162.

[2] Padhy, P., Martinez, K., Riddoch, A., Ong, H. L. R., and Hart, J. K. (2005) Glacial environment monitoring using sensor networks. Proceedings of the Workshop on Real-World Wireless Sensor Networks, Stockholm, Sweden.

[3] Werner-Allen, G., Lorincz, K., Ruiz, M., Marcillo, O., Johnson, J., Lees, J., and Welsh, M. (2006) Deploying a wireless sensor network on an active volcano. IEEE Internet Computing, 10, 18-25.

[4] Chintalapudi, K., Fu, T., Paek, J., Kothari, N., Rangwala, S., Caffrey, J., Govindan, R., Johnson, E., and Masri, S. (2006) Monitoring civil structures with a wireless sensor network. IEEE Internet Computing, 10, 26-34.

[5] Krause, A., Singh, A. P., and Guestrin, C. (2008) Near-optimal sensor placements in Gaussian processes: Theory, efficient algorithms and empirical studies. Journal of Machine Learning Research, 9, 235-284.

[6] Rahman, F. and Shabana, N. (2006) Wireless sensor network based personal health monitoring system. WSEAS Transactions on Systems, 5, 966-972.

[7] Simon, G., Balogh, G., Pap, G., Maroti, M., Kusy, B., Sallai, J., Ledeczi, A., Nadas, A., and Frampton, K. (2004) Sensor network-based countersniper system. Proceedings of the 2nd International Conference on Embedded Networked Sensor Systems, Baltimore, MD, USA, pp. 1-12.

[8] Ledeczi, A. and et al. (2005) Countersniper system for urban warfare. ACM Transactions on Sensor Networks, 1, 153-177.

[9] Bramberger, M., Doblander, A., Maier, A., Rinner, B., and Schwabach, H. (2006) Distributed embedded smart

\footnotetext{
${ }^{9}$ Note the algorithms are not necessarily identical in this case, since the algorithm with flexible routing allows individual nodes to forward data through multiple routes.
} 
cameras for surveillance applications. Journal of IEEE Computer, 39, 68-75.

[10] He, T. and et al. (2006) VigilNet: An integrated sensor network system for energy-efficient surveillance. $A C M$ Transactions on Sensor Networks, 2, 1-38.

[11] Zahariadis, T. and Voliotis, S. (2007) Open issues in wireless visual sensor networking. Proceedings of the 14 th International Workshop on Systems, Signals and Image Processing and the 6th EURASIP Conference focused on Speech and Image Processing, Multimedia Communications and Services, Slovenia, pp. 335-338.

[12] Chalamala, B. R. (2007) Portable electronics and the widening energy gap. Proceedings of the IEEE, 95, 2106-2107.

[13] Kleihorst, R., Schueler, B., Danilin, A., and Heijligers, M. (2006) Smart camera mote with high performance vision system. Proceedings of the ACM SenSys Workshop on Distributed Smart Cameras, Boulder, CO, USA, pp. 17-21.

[14] Ekanayake, V., Kelly-IV, C., and Manohar, R. (2004) An ultra low-power processor for sensor networks. SIGPLAN Notices, 39, 27-36.

[15] Hempstead, M., Tripathi, N., Mauro, P., Wei, G. Y., and Brooks, D. (2005) An ultra low power system architecture for sensor network applications. $A C M$ SIGARCH Computer Architecture News, 33, 208-219.

[16] Meninger, S., Mur-Miranda, J. O., Amirtharajah, R., Chandrakasan, A. P., and Lang, J. H. (2001) Vibrationto-electric energy conversion. IEEE Transactions on Very Large Scale Integration Systems, 9, 64-76.

[17] Raghunathan, V., Kansal, A., Hsu, J., Friedman, J., and Srivastava, M. (2005) Design considerations for solar energy harvesting wireless embedded systems. Proceedings of the 4 th International Symposium on Information Processing in Sensor Networks, Los Angeles, California, USA, pp. 457-462.

[18] Rinner, B., Winkler, T., Schriebl, W., Quaritscha, M., and Wolf, W. (2008) The evolution from single to pervasive smart cameras. Proceedings of the 2nd ACM/IEEE International Conference on Distributed Smart Cameras, Stanford, California, USA, pp. 1-10.

[19] Kho, J., Rogers, A., and Jennings, N. R. (2009) Decentralised control of adaptive sampling in wireless sensor networks. ACM Transactions on Sensor Networks, 5, Article No. 19 (35 pages).

[20] Mathur, G., Desnoyers, P., Ganesan, D., and Shenoy, P. (2006) Ultra-low power data storage for sensor networks. Proceedings of the 5th International Conference on Information Processing in Sensor Networks, Nashville, Tennessee, USA, pp. 374-381.

[21] Sinha, P. and Zoltners, A. A. (1979) The multiplechoice Knapsack problem. Operations Research, 27, 503-515.

[22] Feigenbaum, J., Papadimitriou, C., Sami, R., and Shenker, S. (2005) A BGP-based mechanism for lowestcost routing. Distributed Computing, 18, 61-72.

[23] Padhy, P., Dash, R. K., Martinez, K., and Jennings, N. R. (2006) A utility-based sensing and communication model for a glacial sensor network. Proceedings of the 5th International Conference on Autonomous Agents and Multiagent Systems, Hakodate, Japan, pp. 1353-1360.
[24] Mainland, G., Parkes, D. C., and Welsh, M. (2005) Decentralised, adaptive resource allocation for sensor networks. Proceedings of the 2nd USENIX/ACM Symposium on Networked Systems Design and Implementation, Boston, MA, USA, pp. 315-328.

[25] Hirayama, K. and Yokoo, M. (1997) Distributed partial constraint satisfaction problem. Proceedings of the $3 \mathrm{rd}$ International Conference on Principles and Practice of Constraint Programming, Schloss Hagenberg, Austria, pp. 222-236.

[26] Yokoo, M. and Hirayama, K. (1996) Distributed breakout algorithm for solving distributed constraint satisfaction problems. Proceedings of the 2nd International Conference on Multi-Agent Systems, pp. 401-408.

[27] Modi, P. J., Shen, W. M., Tambe, M., and Yokoo, M. (2005) ADOPT: Asynchronous distributed constraint optimization with quality guarantees. Artificial Intelligence, 161, 149-180.

[28] Petcu, A. and Faltings, B. (2005) DPOP: A scalable method for multiagent constraint optimization. Proceedings of the 19th International Joint Conferences on Artificial Intelligence, Edinburgh, Scotland, pp. 266-271.

[29] Mailler, R. and Lesser, V. (2006) Asynchronous partial overlay: A new algorithm for solving distributed constraint satisfaction problems. Journal of Artificial Intelligence Research, 25, 529-576.

[30] Farinelli, A., Rogers, A., Petcu, A., and Jennings, N. R. (2008) Decentralised coordination of low-power embedded devices using the max-sum algorithm. Proceedings of the 7 th International Conference on Autonomous Agents and Multiagent Systems, Estoril, Portugal, pp. 639-646.

[31] Pizzocaro, D., Johnson, M. P., Rowaihy, H., Chalmers, S., Preece, A., Bar-Noy, A., and Porta, T. L. (2008) A knapsack approach to sensor-mission assignment with uncertain demands. Proceedings of the International Conference on Unmanned/Unattended Sensors and Sensor Networks $V$. 